\title{
QS能
}

\section{LOS PROGRAMAS DE SALUD Y BIENESTAR EN EL TRABAJO COMO PALANCA PARA LA SOSTENIBILIDAD EMPRESARIAL}

La salud de los trabajadores y su bienestar son elementos fundamentales para la productividad y la sostenibilidad de las empresas. En este artículo se reflexiona sobre la respuesta que habría que dar a las amenazas actuales del tejido empresarial, principalmente el absentismo, la baja productividad y el envejecimiento de población trabajadora. A estos retos se suman la cuarta revolución industrial, con la robótica, la industria 4.0 y la economía digital, entre otras.

Palabras clave: trabajadores, productividad, economía digital, industria 4.0.

Clasificación JEL: J28, J58.

\section{El coste de la no prevención}

Hoy, pocos discuten que la inversión en un entorno y una organización del trabajo más seguros y saludables ha conducido a un descenso significativo del número de accidentes y enfermedades profesionales. Ha salvado muchas vidas y evitado un inmenso sufrimiento humano, pero, además, desde el punto de vista económico, también es rentable 1 .

\footnotetext{
* Director del Departamento de Promoción de la Salud y Epidemiología Laboral del Instituto Nacional de Seguridad y Salud en el Trabajo. Ministerio de Trabajo, Migraciones y Seguridad Social. Versión de diciembre de 2019.

DOI: https://doi.org/10.32796/bice.2020.3119.6955
}

1 Así se pone de manifiesto en la Comunicación de la Comisión al Parlamento Europeo, al Consejo, al Comité Económico y Social Europeo y al Comité de las Regiones, relativa a un marco estratégico de la UE, en materia de salud y seguridad en el trabajo 2014-2020 (COM/2014/ 0332 final) (https://eur-lex.europa.eu/legal-content/ES/TXT/?uri=celex\%3A 52014DC0332).
La mejora de la salud y la productividad de los trabajadores constituyen activos estratégicos no solo para las empresas en mercados locales y mundiales, sino también para las sociedades en su conjunto. La seguridad, la salud y el bienestar en el trabajo deben ocupar un lugar preeminente en los programas nacionales e internacionales y conducir a la generación de una cultura genuina de la promoción de la salud, dentro y fuera del centro de trabajo. Para alcanzar ese objetivo es necesario impulsar la movilización de las partes interesadas del sector público, gubernamental, empresarial y de la sociedad civil, a nivel internacional.

Cada vez hay una mayor evidencia de la importancia tanto de realizar actividad física como de reducir el sedentarismo, ya que ambos factores tienen una clara influencia en la mejora de la salud, en la prevención de las enfermedades crónicas y, por tanto, en la $\triangleright$ 
calidad y la esperanza de vida de la población. La práctica habitual de actividad física, de moderada intensidad, aumenta la autoconfianza y mejora el funcionamiento intelectual.

Es este cambio de orientación algo que constituye una tendencia; los informes periódicos de Buck Consultants identifican un interés creciente en promover una cultura de bienestar en la empresa que se gestiona fundamentalmente como parte del valor que se ofrece a los empleados y que se enfoca en su relación con la mejora del engagement, la atracción y retención del talento y la productividad como resultado de este enfoque (Buck Consultant, 2018).

Otra realidad actual es que para una visión holística de la salud ya no basta con contemplar solo los aspectos ligados al medio ambiente de trabajo. Las amenazas al bienestar pueden estar relacionadas, o no, con el trabajo, del mismo modo que los elementos promotores de salud pueden estar vinculados, o no, a aspectos laborales. Unos y otros configuran la salud y bienestar de la población trabajadora, que depende de las condiciones de seguridad, de salud y bienestar de los lugares de trabajo y que modula, en definitiva, la salud y el bienestar de la población en su conjunto (Schulte et al., 2015).

Entre los factores de trabajo asociados a los riesgos psicosociales que pueden generar insatisfacción y falta de bienestar se incluyen: una carga de trabajo y un ritmo de trabajo excesivos, la inseguridad laboral, horarios de trabajo inflexibles, horarios de trabajo irregulares, imprevisibles o socialmente inadecuados, escasas relaciones interpersonales, falta de participación, un papel poco claro en la organización, una escasa comunicación, escasas perspectivas de carrera profesional y exigencias contradictorias entre el trabajo y el $\triangleright$

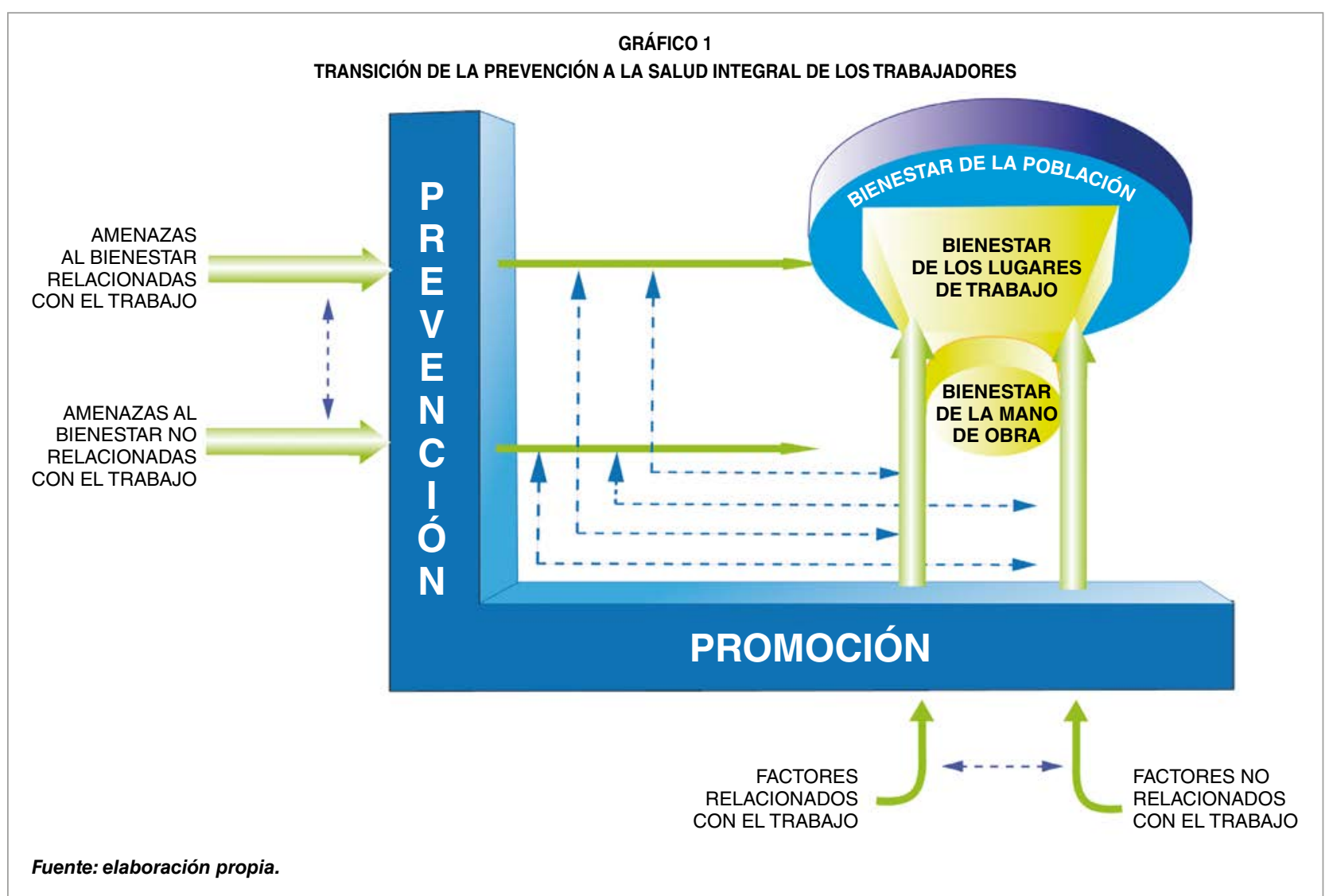


hogar. La Segunda Encuesta Europea de Empresas sobre Riesgos Nuevos y Emergentes (ESENER-2), realizada en 2014 por la Agencia Europea de Seguridad y Salud en el Trabajo (EU-OSHA), es una encuesta multinacional a gran escala dirigida a organizaciones, que abarca 36 países europeos. Algunos datos de esta encuesta invitan al optimismo, ya que el $55 \%$ de los centros de trabajo españoles ha aplicado, en los últimos tres años, alguna medida para prevenir los riesgos psicosociales. Las más habituales son la reorganización del trabajo $(40 \%)$ y el asesoramiento confidencial para trabajadores (31\%). Otras, como la puesta en marcha de un procedimiento de resolución de conflictos y la intervención en caso de demasiadas horas de trabajo u horario irregular, se aplican con menor frecuencia $(27 \%$ y $18 \%$ de los centros, respectivamente) (INSHT, 2015a).

Los costes totales de los trastornos de salud mental en Europa (relacionados y no relacionados con el trabajo) se han estimado en $\mathbf{2 4 0 . 0 0 0}$ millones de euros al año, de los cuales más de la mitad (136.000 millones) se vinculan con la pérdida de productividad, incluido el absentismo ligado a bajas por enfermedad. En este mismo sentido, el informe de 2012, de la Red Europea del Corazón, estimó que el coste de dichas enfermedades para la economía de la UE, en 2009, fue de 196.000 millones de euros. En España, este coste supuso el $8 \%$ del gasto sanitario total (ENWHP, 2009).

No podemos olvidar otras enfermedades, con un alto coste social, como son los trastornos musculoesqueléticos o la diabetes, en las cuales la promoción de la salud tiene también un papel preventivo relevante.

En definitiva, es necesario crear más entornos propicios que protejan la salud física y mental o emocional y que promuevan el comportamiento saludable, utilizando incentivos y desincentivos, medidas reglamentarias y fiscales, leyes y otros tipos de normativas, así como la educación para la salud, con el objetivo último de promover trabajos saludables en todas las edades, lema de la anterior campaña de la Agencia Europea para la Seguridad y la Salud en el Trabajo (EU-OSHA, 2015).

Es obvio que esta tarea no podría llevarse a la práctica sin el firme compromiso de las gerencias de las empresas, de la capacitación de los gestores de recursos humanos, de los líderes y sublíderes que sean capaces de motivar y poner en valor los grandes beneficios que la salud y el bienestar tienen para la empresa, para el trabajador y para la sociedad. Si hace una década se hablaba de globalización, ahora estamos en la era de la «conectividad». La telemática y las redes sociales y empresariales han cambiado la manera no solo de trabajar, también de relacionarse. Esto obliga a que las empresas necesiten contar con líderes que sean capaces de gestionar los talentos de forma más eficiente.

Este enfoque entronca claramente con los objetivos de la responsabilidad social corporativa (RSC). Ya está perfectamente demostrado que invertir en promoción de la salud y bienestar es rentable, pero además se cumple con un compromiso ético de las empresas de contribuir a mejorar el estado de salud y el bienestar, no solo de sus trabajadores, también de sus familias y de la comunidad donde están ubicadas.

Crear un entorno de trabajo seguro y saludable no es fácil, ni tampoco extremadamente difícil. Se puede conseguir el objetivo de «trabajadores sanos, motivados y competentes, en empresas seguras, saludables, socialmente responsables y sostenibles", pero hay que hacerlo poco a poco y de forma secuencial, buscando que las intervenciones concuerden con el $\triangleright$ 
nivel de compromiso de la empresa y con los recursos disponibles.

Los beneficios de una gestión integral de la salud de los trabajadores, más allá de los requerimientos legales, se pueden ver reflejados tanto a nivel individual (trabajador) como a nivel de la organización (empresa), y en beneficio de la sociedad en su conjunto.

Finalmente, está el reto del retorno o vuelta al trabajo de personas con ausencias medias o prolongadas por enfermedad. El absentismo y la falta de rendimiento, y su repercusión en la productividad y competitividad de la empresa, se han convertido últimamente en motivo de preocupación, no tan solo para las empresas, sino también para los países y la sociedad en general, máxime si la ausencia al trabajo acaba con la salida del trabajador del mercado laboral y su acceso a una pensión por incapacidad ${ }^{2}$.

Los trabajadores de más edad presentan un riesgo mayor de jubilación anticipada por incapacidad y de ausencias prolongadas por enfermedad, cuyos costes resultan significativos. Un reciente documento de la Agencia Europea para la Seguridad y Salud en el Trabajo ofrece información actualizada sobre los conocimientos relativos a los sistemas, los programas y las intervenciones en materia de rehabilitación y reincorporación al trabajo y sus distintos componentes. En el documento se analizan las pruebas actuales, en relación con la eficacia de las intervenciones, y se exploran los factores subyacentes a unas experiencias de rehabilitación y de reincorporación al trabajo (EU-OSHA, 2016).

Los programas de vuelta al trabajo (muy poco frecuentes en España) consisten

\footnotetext{
2 Los datos del último informe Adecco sobre absentismo dan una cifra de unos costes directos de más de 5.000 millones de euros, equivalente al $0,4 \%$ del PIB (https://www.adeccoinstitute.es/wp-content/uploads/ 2019/06/VIII-Informe-Absentismo.pdf).
}

principalmente en establecer una serie de acciones en la empresa, en colaboración o no con otros organismos o entidades, para facilitar la rápida reincorporación de los trabajadores de baja por enfermedad o accidente, y en las mejores condiciones.

Los beneficios de estos programas se producen a dos niveles:

- Para el trabajador, ya que mantiene su capacidad de trabajo y su empleo, los ingresos, la autoestima, la estabilidad familiar y los lazos sociales y, obviamente, su salud tanto física como mental y social.

- Para la empresa, en términos de productividad, reducción del absentismo, aumento de la satisfacción y de la motivación de los trabajadores, retención de los trabajadores clave, disminución de los costes de formación o substitución ligados a la rotación involuntaria del personal, mejora de la calidad del producto o servicio, etcétera.

Los principios en los que se basa un buen programa de vuelta al trabajo, siempre desde un enfoque proactivo y de aproximación a las necesidades del trabajador en cuestión, son:

- La intervención precoz.

- La implicación del trabajador y de sus representantes.

- El compromiso de la dirección.

- La existencia de una política integral de salud en la empresa y de la asignación de responsabilidades en el liderazgo y ejecución del programa.

- Unas soluciones a medida y flexibles para las que se pongan a disposición los recursos adecuados. 
En sintonía con el objetivo de la Campaña Europea de "Trabajos saludables en cada edad", basada en el proyecto del Parlamento Europeo titulado «Trabajo más seguro y saludable a cualquier edad", dirigido por la EU-OSHA, el Instituto Nacional de Seguridad y Salud en el Trabajo (INSST) viene desarrollando herramientas para promover hábitos de vida saludables, aportando materiales, infografías, vídeos e informes técnicos que se pueden encontrar en el portal temático sobre Promoción de la Salud, alojado en la página del INSST (INSST, 2019).

\section{2. ¿Cuál es el marco conceptual de la promoción de la salud en los lugares de trabajo (PST)?}

La empresa se responsabiliza de la seguridad y salud de sus trabajadores, pero debe también mejorar la calidad del trabajo, potenciando los recursos para promocionar la calidad de vida, la salud y el bienestar.

Al margen de los ámbitos más propios del individuo, están, también, los de la salud medioambiental, entre los que podemos considerar aquellos propios de los ambientes de trabajo: riesgos biológicos, físicos o químicos, más propios de la prevención de riesgos laborales, pero también los vinculados al medio ambiente extralaboral, a los que la empresa puede contribuir mediante políticas de movilidad, disminuyendo el uso del vehículo privado en los desplazamientos y reduciendo las emisiones de $\mathrm{CO}_{2}$ y mejorando la seguridad vial, o bien implantando sistemas de gestión ambiental, con selección de los residuos urbanos que genera, reciclaje de papel para fotocopias de uso interno, control del consumo de agua y luz, etcétera.

En los últimos años se han globalizado los mercados, se han mejorado los niveles de vida, pero también se han incrementado factores negativos como los hábitos alimentarios inapropiados, la disminución de la actividad física y un mayor consumo de tabaco y otras drogas. Todos ellos, unido al progresivo envejecimiento de la población, como consecuencia de la prolongación de la esperanza de vida y de la mejora de la atención sanitaria y de los procesos asistenciales, han sido factores determinantes del incremento de la prevalencia de enfermedades crónicas, incluidas la obesidad, la diabetes mellitus, las enfermedades cardiovasculares, la hipertensión y los accidentes cerebrovasculares, y algunos tipos de cáncer; causas, cada vez más importantes, de discapacidad y muerte prematura, aparte de suponer una carga adicional para los presupuestos sanitarios nacionales.

Por su parte, el Sistema Nacional de Salud, tradicionalmente, ha centrado su actuación en las personas enfermas, pero el estado del bienestar ha entrado en crisis de sostenibilidad y es necesario desarrollar y reforzar estrategias de actuación en la población sana, de forma que esta actuación preventiva ralentice el deterioro de la salud, mejorando el desempeño de la actividad laboral de una población trabajadora cada vez más añosa.

El lugar de trabajo es un entorno muy apropiado para el desarrollo de actuaciones de promoción y prevención de la salud. En este ámbito surge la Promoción de la Salud en el Trabajo (PST), definida por la Red Europea de Promoción de la Salud en el Trabajo (ENHWP), en su Declaración de Luxemburgo, como «aunar los esfuerzos de los empresarios, los trabajadores y la sociedad para mejorar la salud y el bienestar de las personas en el lugar de trabajo ’ $^{3}$.

3 ENWHP. Luxembourg Declaration on Workplace Health Promotion 1997 (updated april 2018). Recuperado el 4 de diciembre de 2019 (http:// www.move-europe.it/file\%20pdf/2018\%20Version\%20Luxembourg_ Declaration_V2.pdf). 
La PST es una estrategia empresarial moderna que aspira a aumentar la capacidad individual de la población trabajadora para mantener su salud y calidad de vida. Por tanto, la PST debe incluir medidas dirigidas al individuo y al ambiente, que persigan no solo controlar el riesgo derivado de la actividad laboral, sino ir más allá, implantando actuaciones dirigidas a proteger y mejorar la salud de los trabajadores.

La relación entre ciertos factores de riesgo y el incremento del absentismo laboral y el descenso de la productividad de las organizaciones ha sido ampliamente analizada. En la literatura científica se describe que los trabajadores con falta de sueño presentan dos veces más problemas de rendimiento que el resto (Doi et al., 2003), que la mala gestión del estrés ocasiona pérdidas de 5,3 horas por semana y trabajador (Burton et al., 1999), que los trabajadores con sobrepeso o con obesidad presentan mayor número de bajas, o que las personas que no hacen ejercicio reportaban el doble de bajas que las personas que hacían ejercicio al menos una vez por semana (Casajús y Vicente-Rodríguez, 2011).

Aceptemos, por tanto, que las iniciativas saludables reportan beneficios económicos a las organizaciones que las implementan, en términos de incremento de la productividad, competitividad y sostenibilidad de las empresas, y la disminución de los costes sanitarios y de la Seguridad Social. Por cada euro gastado en programas de prevención, salud y bienestar se obtiene un retorno de la inversión promedio de 2,54 euros, aunque este retorno no revierte directamente en el empleador, aunque sí en el sistema (Kreis y Bödeker, 2004; Carpintero et al., 2014).

En este sentido, el INSST ha puesto a disposición de las empresas una herramienta de apoyo para la gestión de los programas de promoción de la salud en el trabajo. Su utilidad tiene un enfoque doble, pues, por una parte, aporta una orientación sobre la calidad metodológica que los programas deben contemplar $y$, por otra, permite realizar una estimación del retorno económico de la inversión realizada en este contexto 4 .

La salud no deja de ser el resultado de la interacción de una serie de factores ligados a la biología humana, a los hábitos de vida y a las creencias y actitudes de la persona, a los entornos laboral y extralaboral, a los servicios de salud y a una serie de factores transversales como el desarrollo económico, tecnológico, social, cultural y demográfico (OMS, 2013).

Algunos de estos factores no pueden controlarse desde la empresa, sin embargo otros sí. La gestión de la salud en el trabajo no deberá, en ningún caso, centrarse en la persona como «causante» de sus males, sino que requerirá de una actuación equilibrada a tres niveles:

- Individuo: mejorando la sensibilización, conocimiento y habilidades de la persona, y promoviendo un cambio de actitudes y comportamiento.

- Entorno: creando entornos físicos y sociales que promuevan buenos hábitos de salud y que permitan elecciones seguras y saludables.

- Organización: integrando los objetivos de salud en los objetivos de la empresa, estableciendo incentivos para reforzar los comportamientos y asignando recursos, entre otras cosas.

\footnotetext{
4 Las definiciones de cada concepto y las asunciones matemáticas se describen en la propia herramienta (https://www.insst.es/aip/-/asset_ publisher/2klzdOfrKZo9/content/herramienta-para-la-valoracion-delos-programas-de-promocion-de-la-salud-en-el-trabajo-ano-2018-encatalogo-?).
} 


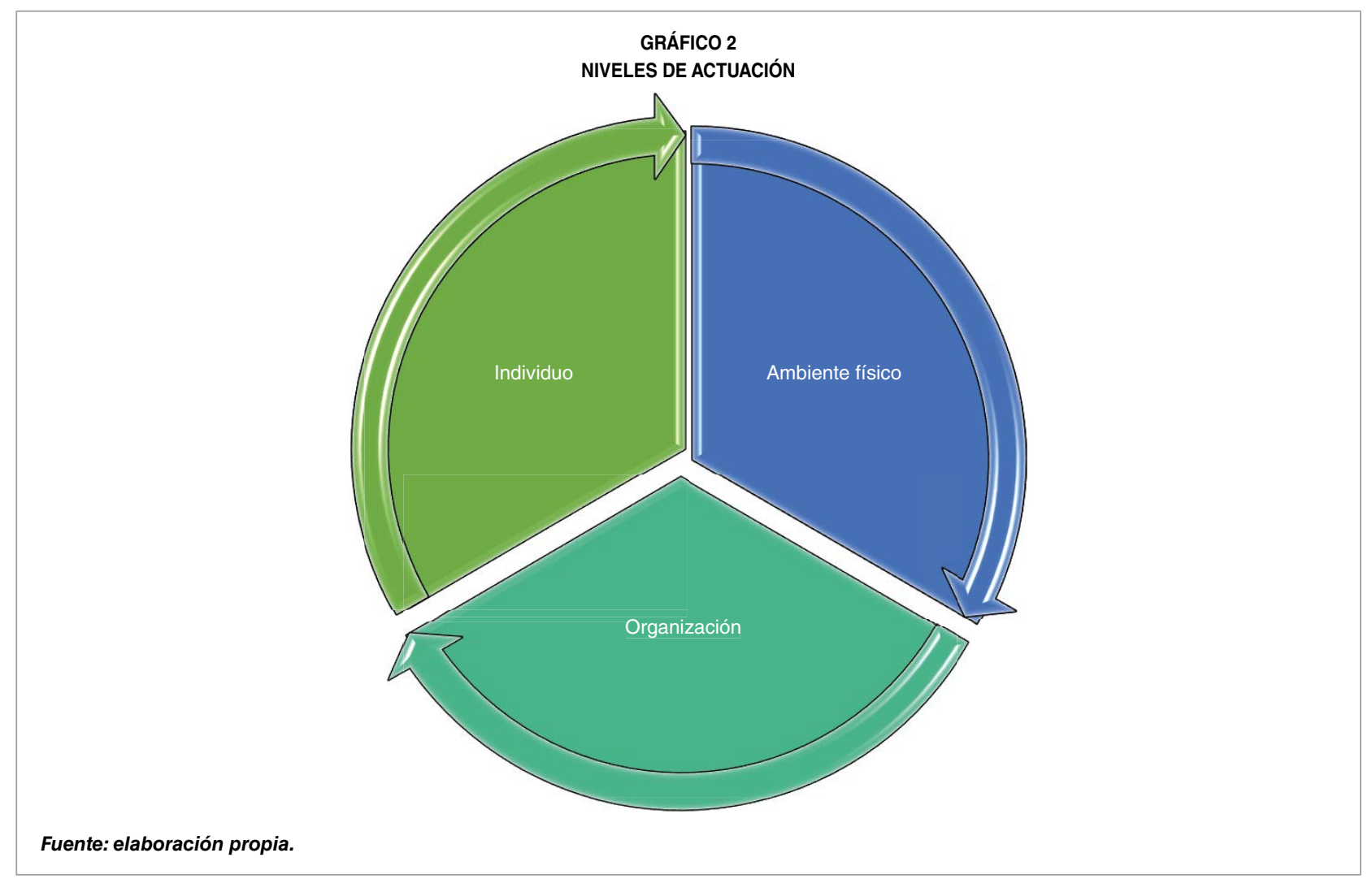

La consecuencia lógica de esta perspectiva es la prioridad de las actuaciones sobre las condiciones estructurales, de la organización y ambientales por encima de las condiciones individuales y de comportamiento. En una organización saludable, el ambiente de trabajo permite al trabajador utilizar plenamente todo su potencial. Esto implica no solo una gestión responsable de los riesgos para la salud y la seguridad (riesgos físicos, biológicos, químicos, psicosociales), sino que debe ser también una posibilidad para el trabajador de desarrollarse y crecer hasta su máximo nivel. Para ello es esencial mantener un equilibrio adecuado en relación con el clima laboral, la formación y el estilo de gestión, así como asegurar una comunicación, unos comportamientos y unas actitudes de apoyo en la organización.

\subsection{La promoción de la salud más allá de la prevención de riesgos}

La prevención de riesgos laborales se refiere al conjunto de actividades aplicadas a los individuos, o grupos de riesgo, para evitar la aparición del daño o mitigar sus efectos. En prevención de riesgos laborales, las intervenciones persiguen eliminar o minimizar los riesgos derivados del trabajo; es decir: los accidentes de trabajo, las enfermedades profesionales y las enfermedades relacionadas con el trabajo.

Prevención del daño y promoción de la salud se utilizan frecuentemente como términos complementarios pese a que frecuentemente se produce una superposición del contenido y de las estrategias. De hecho, en lo tocante a salud laboral, la evolución del concepto de salud ha favorecido la migración desde unas $\triangleright$ 
actividades meramente reactivas, y centradas en los problemas, hacia actividades proactivas, que hacen hincapié en los recursos y los factores positivos.

\subsection{Experiencias en Europa}

A nivel europeo destacaremos que en 1996 nació la Red Europea de Promoción de la Salud en el Trabajo (ENHWP), bajo los auspicios de la Comisión Europea, convirtiendo la promoción de la salud en el lugar de trabajo (PST) en un campo de actuación de la salud pública a nivel nacional y europeo.

EI INSST, como miembro fundador de la ENHWP, puso en marcha, en 2013, el proyecto Red Española de Empresas Saludables con el interés de reconocer el trabajo de las empresas en el ámbito de la mejora de la salud y bienestar de sus trabajadores, así como promover la cultura de la salud, y el intercambio de experiencias empresariales. En el marco de este proyecto se han desarrollado herramientas para evaluar aspectos tales como tabaquismo, actividad física, nutrición, estrés, alimentación, salud mental, gestión de la edad y gestión de las enfermedades crónicas. El proyecto persigue igualmente generar alianzas entre las empresas, Administraciones y organizaciones, públicas y privadas, en torno a estos problemas. Se puede encontrar más información sobre el proyecto de las empresas saludables en el portal temático del INSST ${ }^{5}$.

El informe The Workplace Wellness Alliance (World Economic Forum, 2013) puso de manifiesto que el promedio del retorno de la inversión (ROI) es de 2,54 euros por cada euro

\footnotetext{
5 Anualmente, la red celebra su encuentro donde se presentan los casos de éxito y se reconocen las buenas prácticas empresariales. Estas prácticas también pueden consultarse en la web del INSST (https://www. insst.es/red-espanola-de-empresas-saludables).
}

gastado en programas de bienestar en las empresas. Además, concluyó que el promedio del coste que las empresas pueden recuperar invirtiendo en programas de salud y bienestar es de 545 euros por año.

Sin embargo, no hay consenso sobre los efectos positivos de los programas de salud y bienestar, en términos económicos. La consultora RAND Europa ha desarrollado un modelo económico sujeto a un marco predictivo basado en la identificación de los factores esenciales que impactan en el retorno de la inversión. Usaron datos de una empresa farmacéutica (GSK) así como otras fuentes de información públicas, como el Global Burden of Disease (GBD). Con los datos de GSK el ROI se establece entre 0,26 y 2,12 USD. Financiado por GSK, se publicó un interesante informe que resume la evidencia disponible sobre la efectividad de los programas de salud en el trabajo, subrayando los retos que implica el cálculo del ROI de estas intervenciones (Stepanek et al., 2017).

En España, desde el INSST, como oficina de contacto de la ENWHP, se coordinan las adhesiones a la Declaración de Luxemburgo, a la que ya se han adherido más de ochocientas empresas españolas. Son ya casi cincuenta empresas las que han recibido el reconocimiento a sus buenas prácticas y es creciente el número de candidatas a ese galardón ${ }^{6}$.

\section{3. ¿Es posible la promoción de la salud en las pequeñas y medianas empresas (pymes)?}

Las pymes presentan unas características diferenciales con respecto a las grandes organizaciones:

6 Ejemplos de buenas prácticas saludables en empresas españolas están disponibles en https://www.insst.es/buenas-practicas 
- Menor división del trabajo y estandarización de las tareas y condiciones de trabajo.

- Relaciones más próximas entre empresarios y empleados.

- Mayor grado de flexibilidad.

- Se benefician menos de los servicios de prevención de riesgos laborales.

- La cultura de la organización tiende a dar menos valor al bienestar del empleado y a los recursos humanos en general.

Considerando los limitados recursos financieros de las pequeñas organizaciones, es especialmente difícil para las pymes llevar a cabo actividades de promoción de la salud. Además, el actual conocimiento en PST se basa en experiencias llevadas a cabo en grandes organizaciones que deben ser adaptadas y modificadas en relación a las necesidades de las pymes.

\section{3. ¿Cómo llegar a ser una empresa saludable en la práctica?}

Una empresa que quiera ser reconocida como «saludable» deberá cumplir, ante todo, con la legislación vigente en prevención de riesgos laborales $\mathrm{y}$, a partir de ahí, establecer intervenciones más allá de dicha legislación. Una empresa no puede ser saludable si previamente no es segura, y una empresa solo será sostenible si es segura y saludable.

Si la empresa supera esta primera fase de evaluación, dependiendo del valor obtenido en los cuestionarios de autoevaluación, se le invitará a proseguir hasta alcanzar el reconocimiento de buenas prácticas?

\footnotetext{
7 Para facilitar a las organizaciones una autoevaluación, el INSST pone a disposición de las empresas varias herramientas de análisis y de mejora de sus actividades. Los cuestionarios de autoevaluación están disponibles en la página web https://www.insst.es/-/cuestionarios-deevaluacion-en-pst, en formato en línea y en PDF descargables.
}

Para finalizar cabe comentar que cada año, en torno al mes de mayo, se celebra en el INSST el Encuentro de la Red Española de Empresas Saludables ${ }^{8}$.

\section{Conclusiones y recomendaciones}

Las características de una buena gestión de la salud en el trabajo se pueden resumir en:

- Un diseño sistemático de programas que mejoren la salud del trabajador y de la organización.

- La creación de una cultura de la salud que satisfaga las necesidades tanto de la empresa como del trabajador.

- Una gestión de la salud que se integre en el plan estratégico de la empresa porque es bueno para la salud del trabajador y para la productividad, eficiencia y competitividad de la empresa.

- Una metodología que ayude a las personas a conseguir una salud óptima (emocional, física, social, espiritual e intelectual).

- Una metodología que utilice diversas estrategias para mejorar el conocimiento que sobre la salud tienen los trabajadores y demás actores relevantes y para poner a su disposición un entorno de trabajo que proteja la salud de las personas y que apoye y refuerce las elecciones saludables.

- Promover unas condiciones de trabajo mejoradas, en busca de la calidad y $\triangleright$

8 El último encuentro tuvo lugar el 8 de mayo de 2019, con la entrega de los certificados de buenas prácticas a las empresas reconocidas ese año (https://www.insst.es/-/iv-encuentro-de-la-red-espanola-deempresas-saludables-empresas-seguras-sanas-sostenibles-y-solidariasen-la-nueva-era-digital). 
TABLA 1

DIMENSIONES QUE EVALÚA EL COMITÉ DE VERIFICACIÓN, COORDINADO POR EL INSST

\begin{tabular}{|c|c|}
\hline $\begin{array}{l}\text { 1. El concepto que tiene la } \\
\text { empresa sobre la promoción } \\
\text { de la salud en el trabajo (PST) }\end{array}$ & $\begin{array}{l}\text { Que los programas de PST contemplen el concepto de salud biopsicosocial; actúen de forma } \\
\text { equilibrada sobre la persona, el entorno y la organización; respondan a los valores de justicia } \\
\text { social y poder compartido y se basen en el desarrollo de capacidades individuales y } \\
\text { organizativas. }\end{array}$ \\
\hline 2. Liderazgo & $\begin{array}{l}\text { Las intervenciones para aplicar el programa de PST tendrán el apoyo de la dirección y una } \\
\text { asignación de recursos adecuados (humanos y materiales); debe existir una política o } \\
\text { declaración de PST con implicación expresa de la dirección de la empresa. }\end{array}$ \\
\hline 3. La participación & $\begin{array}{l}\text { Que todos los actores implicados estén integrados en las diferentes fases del proceso, desde } \\
\text { el diseño de la evaluación y que se fomente una toma de decisiones compartida. }\end{array}$ \\
\hline 4. Comunicación & $\begin{array}{l}\text { La comunicación debe ser continuada entre y hacia todos los actores implicados, informando } \\
\text { puntualmente a la dirección y a los trabajadores de los avances y resultados de las } \\
\text { intervenciones. }\end{array}$ \\
\hline 5. Justificación & $\begin{array}{l}\text { Una «buena práctica» en PST debe basarse en un buen conocimiento del contexto y debe } \\
\text { priorizar las actuaciones en función de una evaluación de necesidades. }\end{array}$ \\
\hline 6. Multicomponente & $\begin{array}{l}\text { La intervención debe favorecer una aproximación global, multinivel y positiva de la salud y } \\
\text { debe tener en cuenta las áreas para la acción recomendadas por la OMS. Así mismo, el } \\
\text { proyecto debe integrarse en otros programas internos (PRL, RSC, RR HH, etcétera). }\end{array}$ \\
\hline 7. Planificación & $\begin{array}{l}\text { Los objetivos deben ser coherentes con las necesidades detectadas; los métodos y } \\
\text { herramientas serán adaptados a los objetivos y se debe prever un plan de evaluación y mejora: } \\
\text { el proceso debe ser un proceso continuo. }\end{array}$ \\
\hline 8. Colaboración & $\begin{array}{l}\text { Las personas y los colaboradores, internos y/o externos, se definirán claramente. La acción } \\
\text { llevada a cabo será objeto de un seguimiento continuo por todos los colaboradores. }\end{array}$ \\
\hline
\end{tabular}

la sostenibilidad del trabajo, donde la salud y la seguridad de los trabajadores está asegurada y en las que se cumplen y sobrepasan los requerimientos legales de la normativa vigente.

- Fomentar unos hábitos de vida saludables considerando en las intervenciones cómo puede el entorno de trabajo facilitar y apoyar hábitos, comportamientos y habilidades para una forma de vida más saludable.

- Un entorno facilitador en el que la cultura de la organización refuerza y defiende unos valores éticos que aseguran un trato respetuoso y justo de los trabajadores.

Conseguir el objetivo de trabajadores sanos en empresas saludables debe hacerse de forma paulatina, buscando que las intervenciones concuerden con el nivel de compromiso de la empresa y con los recursos disponibles.

Todo lo anterior ha de interpretarse al margen de los potenciales beneficios económicos de los programas de salud y bienestar, en la idea de que el objetivo final es mejorar la productividad, atraer el talento y avanzar hacia el concepto de valor global de la inversión (VOI), reforzando de manera positiva el valor de lo intangible ${ }^{9}$.

A todo ello se suma el compromiso de España con los Objetivos de Desarrollo del Milenio y de la Agenda 2030, ya que los objetivos 3, 8,10 y 11 están claramente vinculados al $\triangleright$

9 No es casual que todas las empresas que son «top employers» 0 «best place to work» tengan dentro de su estrategia un programa para mejorar el bienestar corporativo de sus colaboradores (http://www. capitalhealth.es/roi-vs-voi/). 
proyecto de Red Española de Empresas Saludables, que tiene por lema promover empresas 4S, seguras, saludables, sostenibles y solidarias e inclusivas ${ }^{10}$.

\section{Bibliografía}

Agencia Europea para la Seguridad y la Salud en el Trabajo (2016). Campaña 2016-2017. Trabajos saludables en todas las edades. Recuperado de https://osha.europa.eu/es/healthy-workplacescampaigns/2016-17-campaign-healthy-workplaces-all-ages

Agencia Europea para la Seguridad y Salud en el Trabajo (2016). Rehabilitation and return to work: Analysis report on EU and Member States policies, strategies and programmes. Recuperado de https://osha.europa.eu/es/publications/rehabilitation-and-return-work-analysis-report-euand-member-states-policies-strategi-0/view

Buck Consultant (2018). Working well. A global survey of workforce wellbeing strategies. Recuperado de https://content.buck.com/hubfs/Downloads/Surveys/Buck\%202018\%20GWS\%20 Executive\%20Summary_final.pdf

Burton, W. N., Conti, D. J., Chen, C. Y., Schultz, A. B., \& Edington, D. W. (1999). The role of health risk factors and disease on worker productivity. Journal of Occupational and Environmental Medicine, 41(10), 863-877.

Carpintero Pérez, P., Lago Antón, S., Neyra Castañeda, A., y Terol Conthe, I. (2014). ¿Es coste-efectivo el desarrollo de programas de promoción de la salud en los lugares de trabajo? Medicina y Seguridad del Trabajo, 60 (236), 566-586. Recuperado de http://scielo.isciii.es/pdf/mesetra/v60n 236/revision2.pdf

Casajús, J. A., y Vicente-Rodríguez, G. (2011). Ejercicio físico y salud en poblaciones especiales.

10 Para leer más sobre los ODS y la Agenda 2030, consúltese https:// www.agenda2030.gob.es/es/objetivos
Exernet. Recuperado de http://www.munideporte.com/imagenes/documentacion/ficheros/ 035D0D7E.pdf

Doi, Y., Minowa, M., \& Tango, T. (2003). Impact and Correlates of Poor Sleep Quality in Japanese White-Collar Employees. Sleep, 26(4), 467-471.

ENWHP (2009). A guide to the business case for mental health. Recuperado de https://www. enwhp.org/resources/toolip/doc/2018/04/23/ mentalhealth_broschuere_businesscase.pdf

Instituto Nacional de Seguridad e Higiene en el Trabajo (2015). Factores de riesgo de enfermedades cardiovasculares en la población trabajadora según la Encuesta Nacional de Salud 2011/2012. Recuperado de https://www.insst.es/ documents/94886/514312/Riesgo+cardiovascular/4f176ab6-5da8-4b21-a2e2-d12860db1414

Instituto Nacional de Seguridad y Salud en el Trabajo (2019). Portal de Promoción de la Salud. Recuperado de https://www.insst.es/promocion-dela-salud

Kreis, J., \& Bödeker, W. (2004). Health-related and economic benefits of workplace health promotion and prevention. Summary of the scientific evidence. BKK Bundesverband. Recuperado de https://www.enwhp.org/resources/toolip/doc/ 2018/04/24/iga-report_3_english.pdf

Organización Mundial de la Salud (2013). Ambientes de trabajo saludables: un modelo para la acción: para empleadores, trabajadores, autoridades normativas y profesionales. Recuperado de http://www.who.int/phe/publications/healthy_ workplaces/es/

Schulte, P. A., Guerin, R. J., Schill, A. L., Bhattacharya, A., Cunningham, T. R., Pandalai, S. P., \& Stephenson, C. M. (2015). Considerations for incorporating "well-being" in public policy for workers and workplaces. American Journal of Public Health, 105(8), e31-e44.

Stepanek, M., Hafner, M., Taylor, J., GrandClement, S., \& Van Stolk, C. (2017).The return of investment for preventive healthcare $D$ 
Francisco Marqués Marqués

programmes. A calculation framework for GSK's Partnership for Prevention (P4P). Recuperado de https://www.rand.org/pubs/research_reports/ RR1787.html
World Economic Forum (2013). The Workplace Wellness Alliance Making the Right Investment: Employee Health and the Power of Metrics. Recuperado de www.bit.ly/1Z7EkOO 